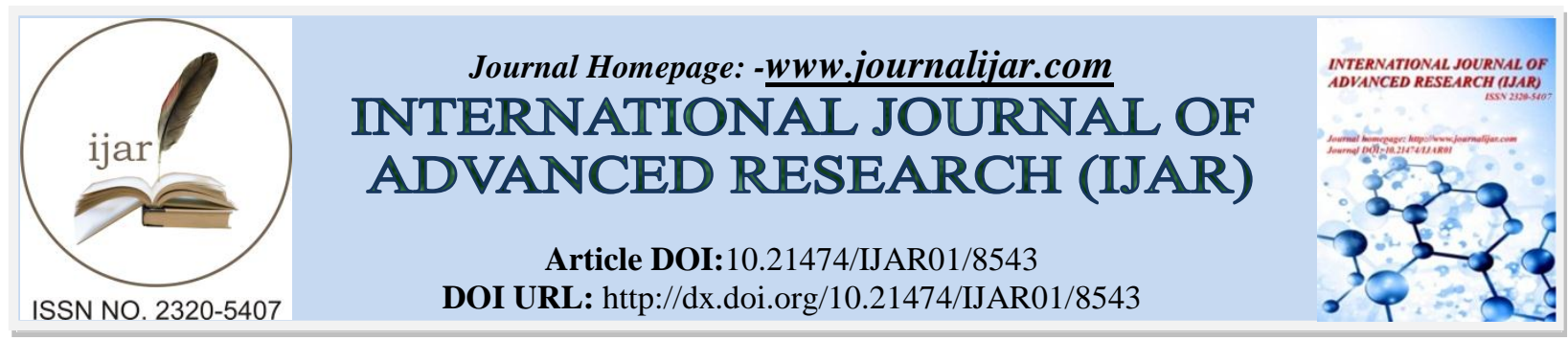

RESEARCH ARTICLE

\title{
STUDY OF EARLY AND LATE ONSET COMPLICATIONS OF TRANSSPHENOIDAL PITUITARY ADENOMA RESECTION: AN INSTITUITIONAL EXPERIENCE.
}

\author{
Mayankkumar, V.D Sinha and AchalSharma.
}

\section{Manuscript Info}

\section{Manuscript History}

Received: 11 December 2018

Final Accepted: 13 January 2019

Published: February 2019

\section{Key words:-}

Transsphenoidal surgery, Pituitary adenoma surgery, Complication of pituitary surgery.

\begin{abstract}
Introduction:Considering the unique anatomical location and important role of pituitary gland in regulation of various endocrine functions, various postoperative complications are expected resulting from surgery on pituitary tumor. In this study, we analyzed various complications of transsphenoidal pituitary surgery and factors affecting it.

Material and method:Study was conducted between September 2015 to November 2016 on a total of 60 patients. All clinical and surgical data were collected regarding tumor size, symptoms, and residual tumor after surgery, functional remission, symptom relief, and complications. All patients underwent neurological, ophthalmological, and endocrinological examinations before and after resection.

Result:Out of 60 patients 43 were operated endoscopically and 17 were operated microscopically. Perioperatively, arachnoid tear was present in $20(33.33 \%)$ patients. $23(38.34 \%)$ cases were having total resection post-operatively, 20(33.33\%) cases were having subtotal resection and in $17(28.33 \%)$ cases either partial resection done or biopsy was taken. Grade of tumor resection was significantly associated with preoperative extent of tumor (p-value-0.003). CSF -leak was associated with Perioperative arachnoid tear significantly ( $p$ - value $<0.001$ ).

Conclusion:Some complications are there in immediate post operative period, but on follow up, both endoscopic and microscopic procedures have fewer side effects and there was no statistical significant difference in complication rate in both types of surgery.
\end{abstract}

Copy Right, IJAR, 2019,. All rights reserved.

\section{Introduction:-}

Pituitary adenomas, with an incidence of almost $25 \%$ in unselected autopsy series ${ }^{[1]}$, account for 10 to $15 \%$ of all intracranial tumors ${ }^{[2]}$ and remain in many cases undiagnosed since they may not cause clinical symptoms. Despite advances in the pharmacological and radio therapeutic management, especially of hormonally active pituitary adenomas, surgery remains the treatment of choice for the majority of these tumors and the gold standard for hormonally inactive adenomas.

Postoperative complications are of major concern in patient with intracranial lesion because it leads to a significant increase in morbidity and mortality. ${ }^{[3]}$ As compared to craniotomy, transsphenoidal surgery offers the advantage of low morbidity and mortality, preservation of normal pituitary function, lower incidence of permanent diabetes

Corresponding Author:-Mayankkumar. 
insipidus, lesser trauma to the frontal lobes and optic chiasm, less blood loss and no external scar. ${ }^{[4]}$ Despite it, there are few complications with transsphenoidal surgery which should be taken care of during and after surgery. Common postoperative complications include hydro-electrolyte and acid-base disorders, diabetes insipidus, visual field alteration, CSF fistula, nasal obstruction, ophthalmoplegia, headache, limb paresis, meningitis, sepsis and/or hospital infection. Other major complications are carotid artery injury and death.

In this study, we aimed to analyze the surgical outcomes and complications in a series of 60 consecutive patients of pituitary adenoma who were operated by transsphenoidal approaches.

\section{Material and Method:-}

The study was conducted on the patients admitted to the Department of Neurosurgery, Sawai Man Singh Medical College, Jaipur, between September 2015 to November 2016, with clinical and radiological diagnosis of sellarsuprasellar mass, of which only those with histopathological diagnosis of pituitary adenoma are included. The patients were enrolled in the study after obtaining an informed and written consent. A total of 60 patients were included in the study. All clinical and surgical data were collected regarding tumor size, symptoms, and residual tumor after surgery, functional remission, symptom relief, and complications. All patients underwent neurological, ophthalmological, and endocrinological examinations before and after resection.

Inclusion criteria are:

Sellarsuprasellar mass confirmed histologically to be pituitary adenoma

\section{Exclusion criteria are:}

Sellar tumor with large parasellar or retrosellar extension

Patients with symptomatic nonfunctional adenoma and all functional adenomas except prolactinoma were submitted to surgery. Surgery for prolactinoma were performed in cases of chiasm compression, failed hormonal control with medical therapy, side effects of medical therapy and refusal of medical therapy by patient. Only patients operated by transsphenoidal route were included in the study. Transsphenoidal surgery were done by either of these approaches i.e., transsphenoidaltransnasal microscopic approach, transsphenoidaltransnasal endoscopic approach, endoscopic assisted microscopic approach or sub labial transsphenoidal microscopic approach.

CT- sella was done on $3^{\text {rd }}$ post operative day. Post operative MRI brain was done at 6 weeks. Gross-total resection (GTR) was assigned when the MRI scan showed no residual tumor, while near total resection (NTR) was attributed to the removal of $\geq 90 \%$, subtotal resection (STR) with a tumor reduction from $70 \%$ to $89.9 \%$ and partial resection (PR) in the case of $\leq 70 \%$ of tumor resection. ${ }^{[5]}$ This was based on preoperative and postoperative volumetric analysis on MRI.

Post operative hormone level was done on $3^{\text {rd }}$ post operative day. Normal range of various hormones were taken as: T3-1.4-4.2pg/ml, T4-0.89-1.76 microgram /dl, T.S.H-0.35-5.5 micro-IU/ml, Serum-Prolactin-1.9-25 microgram/ml, Serum Cortisol-4.3-22.4 microgram/dl and G.H- In female up to 10 nanogram $/ \mathrm{ml}$ and in male up to $1 \mathrm{nanogram} / \mathrm{ml}$. All blood samples for hormone level were taken at 8:00am. Replacement therapy for hormone deficiency was initiated based on post operative endocrinal testing.

Patients were followed for various complications at 48 hours, at $<3$ weeks and $>3$ weeks. Tumors were classified according to their extent, as purely intrasellar entity, sellar with suprasellar extension, sellar with parasellar extension and sellar with retrosellar extension. In this study pituitary adenoma with retrosellar and large parasellar extension were excluded.

Ethical committee clearance was taken beforehand.

\section{Statistical Analysis:-}

Statistical analysis was performed with the SPSS, Trial version 23 for Windows statistical software package (SPSS inc., Chicago, il, USA) and Primer. The Categorical data were presented as numbers (percentage) and were compared among groups using Chi square test. Groups were presented as mean and standard deviation and were compared using by students t-test. $\mathrm{P}$ value $<0.05$ was considered statistically significant. 


\section{Result:-}

A total of 60 consecutive patients were operated by trans-sphenoidal approach for pituitary adenomas. The age of this cohort ranged from 17 to 72 years (mean: 45 years). Of all these patients, 42(70\%) were having non-functional adenomas, 9(15\%) were having features of prolactinoma and $12(20 \%)$ were acromegalic. None of the patients presented with features of ACTH secreting and TSH secreting tumors in our cohort. There were two cases of microadenoma and 58 cases of macro-adenoma. Out of 58 macro-adenoma, 30 cases were with suprasellar extension only and 28 were with suprasellar and parasellar extension. Most common presenting complaint was headache followed by visual disturbances.

Out of 60 patients 43 were operated endoscopically and 17 were operated microscopically. Perioperatively, arachnoid tear was present in 20(33.33\%) patients. In all except 2 patients, fat and fibrin glue was used for sellar floor packing. In 13 patients, fascia-lata was used and in one case a pedicledseptal flap (hadad flap) was used for packing along with fat and fibrin glue. 23(38.34\%) cases were having total resection post-operatively, 20(33.33\%) cases were having subtotal resection and in $17(28.33 \%)$ cases either partial resection done or biopsy was taken. Patients having intra-sellar tumor only, were having $100 \%$ total resection while those with parasellar extension were having only $17.86 \%$ of total resection. Thus, grade of tumor resection was significantly associated with preoperative extent of tumor (p-value-0.003) (see table-1).

Table 1:-Showing Association of extent of tumor to grade of resection

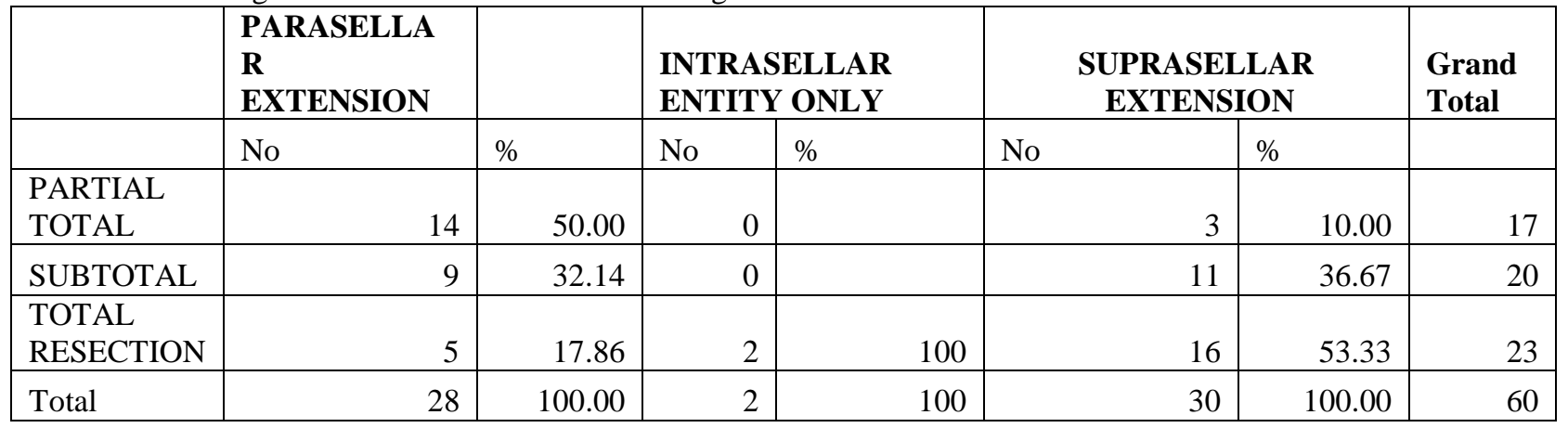

Chi-square $=16.351$ with 4 degrees of freedom; $\quad P=0.003 S$

Pre operatively, Vision was low in 32 cases, out of which it improved in 23(71.87\%) cases while remained same in 8 cases. In one case, vision detoriated which may be due to injury to optic- pathway.

$91.66 \%$ of $\mathrm{GH}$ secreting tumors and $66.66 \%$ of prolactin secreting tumors has complete hormonal remission during follow-up.

Headache improved in $68.9 \%$ patients in immediate post- operative period. At 3 weeks follow up, headache was only in 4 patients. CSF -leak was second most common complication which was associated with Perioperative arachnoid tear significantly ( $\mathrm{p}$ - value <0.001) (see table-2). 14 case were managed conservatively with acetazolamide and bed rest. In 13 cases, lumbar drain was inserted and two cases were re-operated and reconstruction of sellar floor done with fibrin-glue, fat and fascia- lata. At 3 weeks follow up, none of them were having CSF- leak.Diabetesinsipidus was present in 10(16.67\%) cases but all were transient. 7 patients were having $\mathrm{ICH}$ in post operative scan which improved in 4 cases follow up. Two patients were re-operated and one havingassociateddyselectrolytemia. 
Table-2-showing association of post operative CSF leak with type of tumor, Perioperative arachnoid-tear, grade of tumor resection and extent of tumor

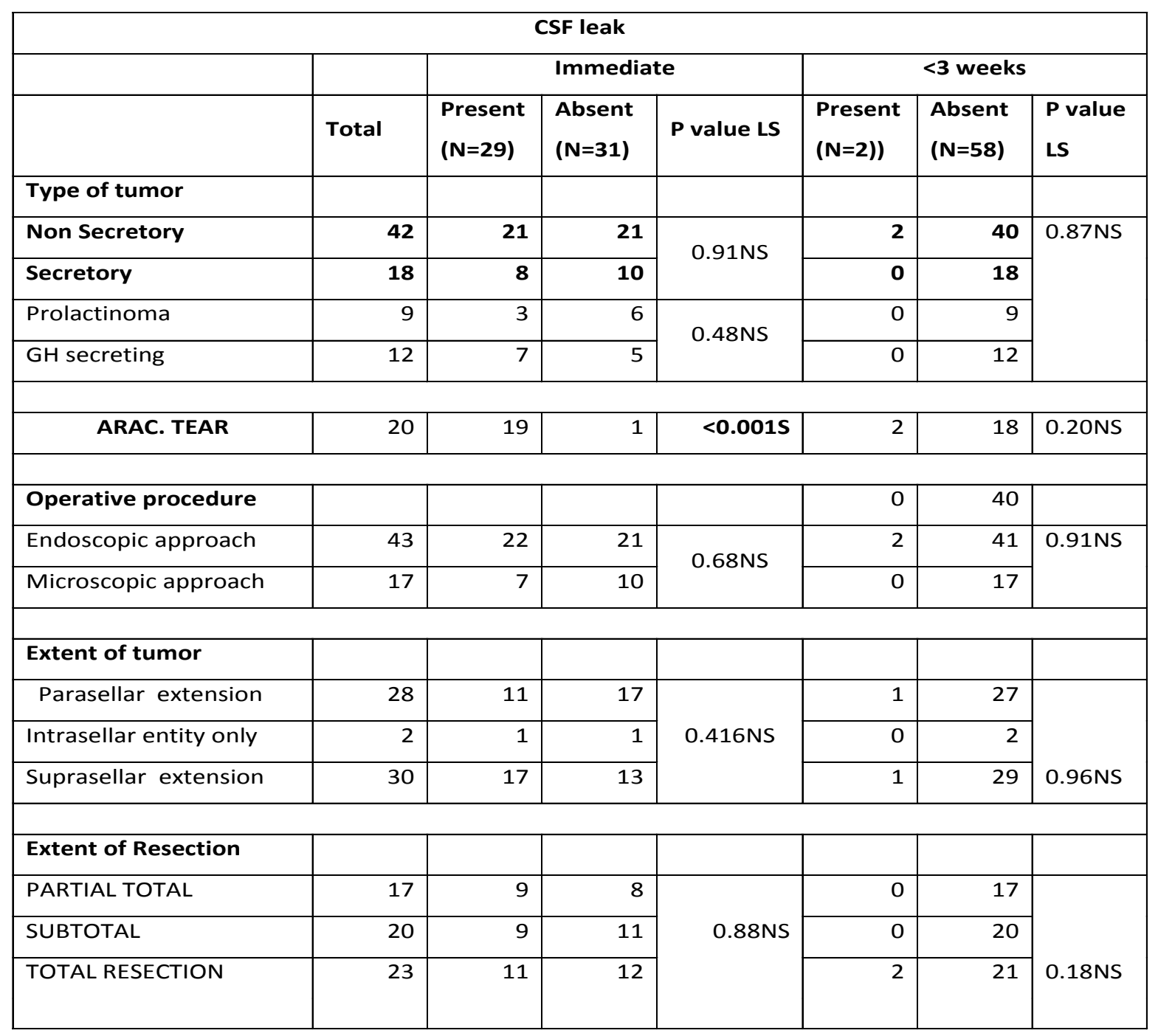


Chart 1:-showing various post operative complications at 48 hours, $<3$ weeks and at >3weeks

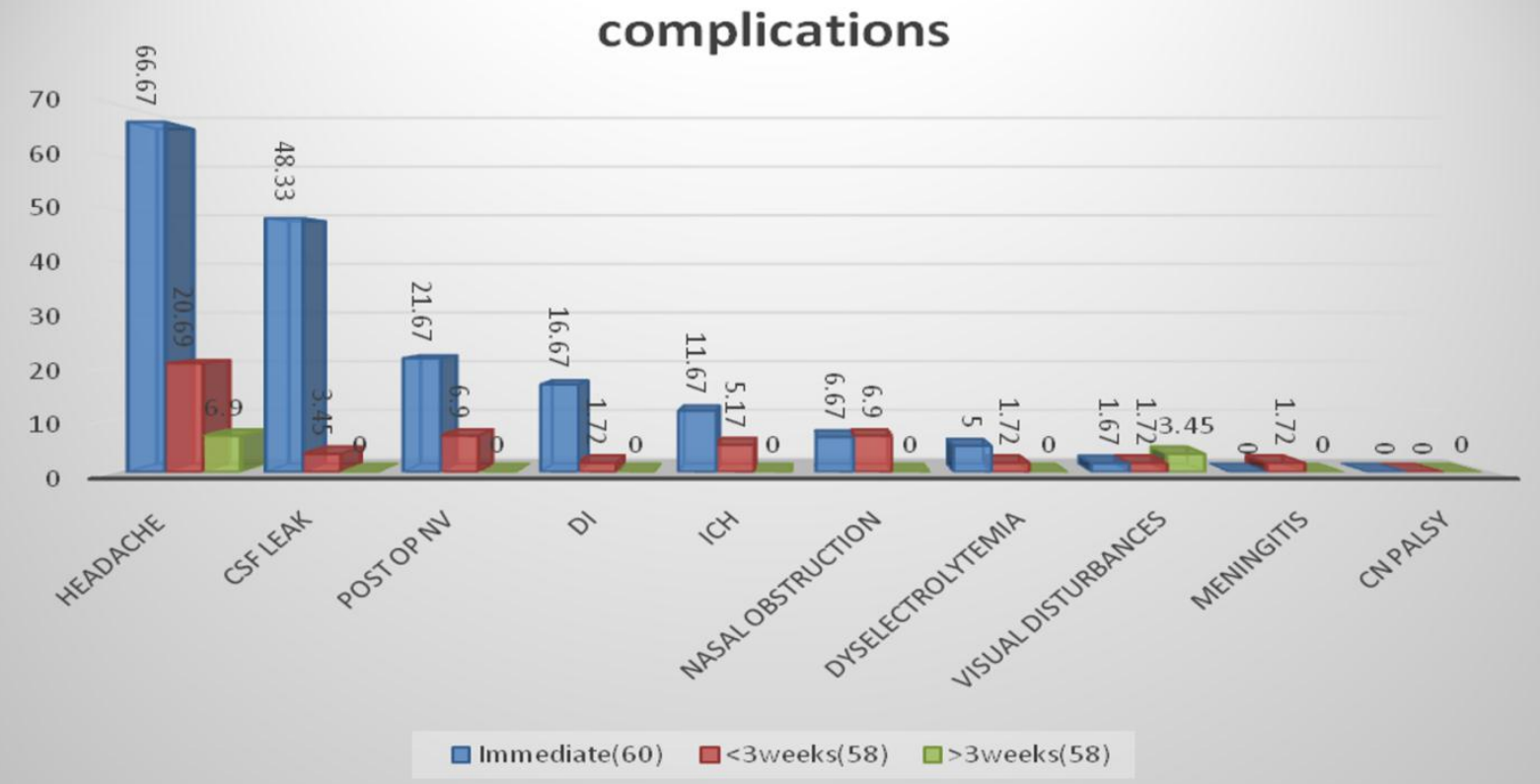

Four cases were re-operated, two because of residual tumor and two for CSF leak. There was two mortality (3.33\%) in our study .There was no case of intra -operative carotid artery injury in our study.

On comparative study of complications in both endoscopic and microscopic approach, no statistically significant difference was found between two groups.

Table-3- Showing postoperative complications in both endoscopic and microscopic transsphenoidal surgery (TSS).

\begin{tabular}{|l|r|r|r|r|r|l|}
\hline & \multicolumn{2}{|l|}{$\begin{array}{l}\text { Endoscopic } \\
\text { TSS(N=43) }\end{array}$} & \multicolumn{2}{c|}{ Microscopic } & & \\
\hline Complications & & \multicolumn{1}{|c|}{$\%$} & & $\%$ & Total & P-value \\
\hline Headache & 30 & 69.76 & 10 & 58.82 & 40 & $0.61 \mathrm{NS}$ \\
\hline PONV & 11 & 25.58 & 3 & 17.65 & 13 & $0.75 \mathrm{NS}$ \\
\hline CSF Leak & 22 & 51.16 & 7 & 41.18 & 29 & $0.68 \mathrm{NS}$ \\
\hline Meningitis & 1 & 2.32 & & 0.00 & 1 & $0.62 \mathrm{NS}$ \\
\hline Dyselectrolytemia & 5 & 11.62 & & 0.00 & 3 & $0.34 \mathrm{NS}$ \\
\hline DI & 9 & 20.93 & 1 & 5.88 & 10 & $0.3 \mathrm{NS}$ \\
\hline ICH & 5 & 11.62 & 2 & 11.76 & 7 & $0.66 \mathrm{NS}$ \\
\hline Visual disturbances & 1 & 2.32 & & 0.00 & 1 & $0.62 \mathrm{NS}$ \\
\hline Cranial nerve Palsy & 0 & 0.00 & 0 & 0.00 & 0 & NA \\
\hline Nasal obstruction & 4 & 9.30 & 1 & 5.88 & 5 & $0.93 \mathrm{NS}$ \\
\hline
\end{tabular}

PONV-postoperative nausea and vomiting, DI-diabetes insipidus ,ICH- intrcerebral hemorrhage, TSS-

transsphenoidal surgery 


\section{Discussion:-}

The basic principles of surgical management of tumors involving the pituitary gland include relieving the pressure on neighboring structures, particularly the visual structures, normalization of hypersecretion, preservation or restoration of pituitary function, prevention of tumor recurrence and collection of tissue for pathological and functional study. ${ }^{[6]}$

In our study only two cases were micro adenoma and rest were macro adenoma with suprasellar and parasellar extensions. This may be due to poverty, illiteracy and poor health awareness among people and lack of health facilities in this part of world. Most of them were giant pituitary tumors.Complete resection is often a technical challenge in case of giant pituitary tumors, even for experienced neurosurgeons, considering that this subgroup of tumors may present with a high degree of invasion of neurovascular structures and large areas of supra- and parasellar extension. ${ }^{[7]}$ In this way, the primary objective of surgery includes relief of mass effect by obtaining $\underset{[8]}{\operatorname{maximal}}$ tumor resection in order to decompress visual pathways, neurovascular structures and the pituitary gland.

At our centre, only 23 (38.34\%) patients were having total resection which is lesser in comparison to other studies. The surgery performed in our patients involved neurosurgeons with variable experience. We have here different learning curves included into this study. This may be the reason for higher complication rate and less complete resection at our centre.In a study by Halvorsen et al, Multivariate analyses showed that the overall risk for complication increased if surgery is performed by a low-volume surgeon. ${ }^{[9]}$ Also, as most of tumor were macro adenoma and giant pituitary adenoma in our study, total resection rate low. According to Goel et al, The rate of gross and near-total tumor removal after transsphenoidal, trans-cranial, or combined procedures ranges from $14.7 \%$ to $74 \%$ in cases of giant pituitary adenomas. ${ }^{[10]}$

There was statistically significant association of preoperative extent of tumor and post operative resection rate, in our study. This was in accordance with Sinha et al. According to them, for patient with preoperative cavernous sinus invasion (CSI), the resection rate was significantly lower than those without CSI. ${ }^{[1]}$

In our study, the second most frequent post-operative complication after headache was CSF leakage. 20 patients presented some degree of CSF leak during surgery and overall 29 patients with post operative CSF leak. As most of our cases were macro adenomas, such a high percentage of CSF leak is expected. In the study by Prabhakar et al, CSF leak was present in $40 \%$ of patients post-operatively. ${ }^{[12]}$ In most cases, the occurrence of the leaks was associated with large macro adenomas with suprasellar extension, which required the surgeon to work in close proximity to the diaphragm sella. Post operative CSF leak and perioperative arachnoid tear are having statistically significant association ( $\mathrm{p}$-value-<0.05) in our study. Shileyet al, also concluded that presence of an intra operative leak were independent predictors of a postoperative leak. ${ }^{[13]}$

The prevalence of diabetes insipidusafter surgery using the endonasal endoscopic technique has been described as $15 \%{ }^{[14]}$ as occurred with $16.66 \%$ of the cases operated in our study. Most cases were transient type and improved at follow up.

Post operative complete hormone remission in prolactinoma was $66.66 \%$ and $91.66 \%$ with $\mathrm{GH}$ secreting tumors. This is in accordance, with study by Chone et al. In their study, hormonal remission was achieved in $87 \%$ of patients during their follow-up. ${ }^{[15]}$

There were two deaths (3.33\%) during the postoperative period of patients with macro adenoma and large extensions, but it was not possible to obtain postmortem examinations to clarify the cause. However, the cause was suspected to be related to surgical management.

On comparing complications in both endoscopic and microscopic procedure, no statistical significant difference was found. Similar results were found in literature also. ${ }^{[9]}$

As only 3 patients were using dopamine agonists (cabergoline) and one each was known diabetic and hypertensive and was on oral antihypertensive drug and oral anti hyperglycemic drug, no statistical study was possible. Further study with bigger cohort is needed to study effect of various drugs on post operative complications. 
In general, a continuous improvement in surgical outcome by increasing one's experience has been documented in a single neurosurgeon's series in pituitary adenomas. ${ }^{[16]}$ Learning curve may be an important factor related to the surgical outcome of transsphenoidal approach by average surgeons.

\section{Conclusion:-}

In our study, we came to conclusion that, though transsphenoidal pituitary adenoma surgery has got some complications in immediate post operative period, there is significant improvement on follow-up. Also, the outcome is going to improve further as the experience of operating surgeons with endoscopic surgery Increases.

\section{Limitations-}

The data were largely descriptive and it is a non randomized, single institutional study. Also, due to a small cohort of patients, statistical analysis could not be performed between certain groups. The surgery performed in our patients involved neurosurgeons with variable experience. Lastly, Long term follow-ups for outcome of these patients were not carried out.

\section{Disclosure of conflict of interest-}

The author had no personal financial or institutional interest in any of the drugs, material or devices described in this article, no financial support received in the generation of this submission.

\section{References:-}

1. Burrow GN, Wortzman G, Rewcastle NB, Holgate RC, Kovacs K, 1981 Microadenomas of the pituitary and abnormal sellar tomograms in an unselected autopsy series. N Engl J Med 304: 156-158

2. Asa SL, 1989 Tumors of the pituitary gland. In: third series (ed) Atlas of tumor pathology, Armed Forces Institute of Pathology, Washington DC: Fascicle 22.

3. Manninen PH, Raman SK, Boyle K, el-Beheiry H. Early postoperative complications following neurosurgical procedures. Can J Anesth 1999; 46:7-14.

4. Lecleraq TA, Griooli F. Avoidance of diabetes insipidus in transsphenoidalhypohysectomy: A modified technique of selective hypophysecotomy. J Neurosurg 1983;58:682-84.

5. Constantino ER, Leal R, Ferreira CC, Acioly MA, Landeiro J. A Surgical outcomes of the endoscopic endonasaltranssphenoidal approach for large and giant pituitary adenomas: Institutional experience with special attention to approach-related complications.ArqNeuropsiquiatr. 2016 May; 74(5): 388-95

6. Maartens NE, Kaye AH. Role of transcranialaproachs in the treatment of sellar and suprasellar lesions. In: Laws Jr. ER, Sheehan JP (Eds). Pituitary surgery - A modern approach. Frontier of Hormone Research. v. 24. Basel: Karger 2006. p. 1-28.

7. Cusimano MD, Kan P, Nassiri F, Anderson J, Goguen J, Vanek I, et al. Outcomes of surgically treated giant pituitary tumours. Can J Neurol Sci. 2012;39(4):446-57. doi:10.1017/S0317167100013950

8. Cappabianca P, Cavallo LM, de Divitiis O, de Angelis M, Chiaramonte C, Solari D. Endoscopic Endonasal Extended Approaches for the Management of Large Pituitary Adenomas. NeurosurgClin N Am. 2015;26(3):323-31. doi:10.1016/j.nec.2015.03.007

9. Surgical complications after transsphenoidal microscopic and endoscopic surgery for pituitary adenoma: a consecutive series of 506 procedures. Halvorsen H, Ramm-Pettersen J, Josefsen R, Rønning P, Reinlie S, Meling T, Berg-Johnsen J, Bollerslev J, Helseth E. ActaNeurochir(Wien). 2014 Mar; 156(3):441-9

10. Goel A, Nadkarni T, Muzumdar D, Desai K, Phalke U, Sharma P: Giant

11. pituitary tumors: a study based on surgical treatment of 118 cases. SurgNeurol 2004, 61:436-445.

12. Sinha S, Sharma BS: Giant pituitary adenomas-an enigma revisited. Microsurgical treatment strategies and outcome in a series of 250 patients. Br J Neurosurg 2010, 24:31-39.

13. Chowdhury T, Prabhakar H, Bithal PK, Schaller B, Dash HH Immediate postoperative complications in transsphenoidal pituitary surgery: A prospective study. .Saudi J Anaesth. 2014 Jul;8(3):335-41

14. Shiley SG, Limonadi F, Delashaw JB, Barnwell SL, Andersen PE, Hwang PH, et al. Incidence, etiology, and management of cerebrospinal fluid leaks following transsphenoidal surgery. Laryngoscope 2003; 113:1283-8

15. Anagnostis P, Adamidou F, Polyzos SA, Efstathiadou Z, Panagiotou A, Kita M. Non-functioning pituitary adenomas: a single center experience. ExpClinEndocrinol Diabetes 2011; 119:314-319

16. Carlos Takahiro Chonea et al:Endoscopicendonasaltranssphenoidal resection of pituitary adenomas: preliminary evaluation of consecutive cases , Braz J Otorhinolaryngol. 2014; 80(2):146-151

17. Nomikos P, Buchfelder M, Fahlbusch R: The outcome of surgery in 668 patients with acromegaly using current criteria of biochemical 'cure'. Eur J Endocrinol 152: 379-387, 2005. 